\section{Andes EDIATRICA \\ Revista Chilena de Pediatría}

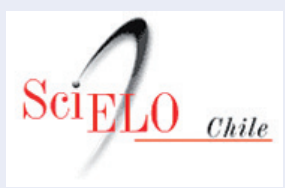

www.scielo.cl

\title{
Diagnóstico prenatal y manejo neonatal precoz de pacientes con Síndrome de corazón izquierdo hipoplásico: experiencia de un centro perinatal
}

\section{Prenatal diagnosis and early neonatal management of patients with hypoplastic left heart syndrome: experience of a perinatal center}

\author{
María Paz Cubillos Celiss, ${ }^{\mathrm{a}, \mathrm{b}}$, Andrea Maccioni Romero ${ }^{\mathrm{a}, \mathrm{c}}$, Constanza Galleguillos Galindo ${ }^{\mathrm{d}}$, \\ Claudia Garrido Sepúlveda ${ }^{\mathrm{e}}$, Carolina Martinovic ${ }^{\mathrm{f}}$, Paula Vargas Innocentif ${ }^{\mathrm{f} g}$
}

\author{
aServicio de Neonatología, Complejo Asistencial Dr. Sótero del Río. Santiago, Chile \\ bDepartamento de Obstetricia y Ginecología, Campus Oriente, Facultad de Medicina de la Universidad de Chile. Santiago, Chile \\ 'Departamento de Neonatología, División de Pediatría, Pontificia Universidad Católica de Chile. Santiago, Chile \\ ¿Servicio de Cardiología Infantil, Complejo Asistencial Dr. Sótero del Río. Santiago, Chile \\ eServicio de Neonatología, Hospital Clínico de la Universidad Católica de Chile. Santiago, Chile

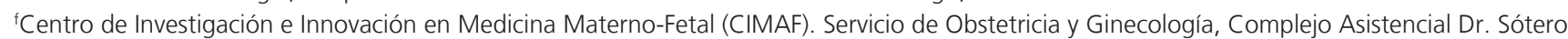 \\ del Río. Santiago, Chile \\ 9División de Obstetricia y Ginecología, Pontificia Universidad Católica de Chile. Santiago, Chile
}

Recibido: 20 de junio de 2020; Aceptado: 25 de febrero de 2021

\section{¿Qué se sabe del tema que trata este estudio?}

El SCIH representa el 2-3\% de todas las cardiopatías congénitas y sin tratamiento quirúrgico su evolución es fatal. La morbimortalidad es elevada, con sobrevida a los 5 años de vida de un 50-57\%. Existen pocos reportes nacionales sobre su manejo neonatal.

\section{¿Qué aporta este estudio a lo ya conocido?}

Este estudio muestra la experiencia de una Unidad de Neonatología en la evaluación prenatal y neonatal de pacientes con $\mathrm{SCIH}$, los que requieren de apoyo hemodinámico y ventilatorio para mantener condiciones adecuadas para la cirugía.

\section{Resumen}

El Síndrome de Corazón Izquierdo Hipoplásico (SCIH) es la forma más común de ventrículo único. A pesar de los avances médico-quirúrgicos, la morbimortalidad se mantiene elevada, especialmente alrededor de la primera cirugía en el período neonatal. Objetivo: Describir las características clínicas de recién nacidos con $\mathrm{SCIH}$, atendidos en un Centro de Derivación perinatal. Pacientes y Método: Estudio retrospectivo descriptivo de todos los recién nacidos con diagnóstico pre y/o postnatal de SCIH entre 2014-2019 en el Complejo Asistencial Dr. Sótero del Río. Se revisaron los registros médicos recolectando las variables demográficas, perinatales, evolución clínica, letalidad
Palabras clave:

Cardiopatías

Congénitas;

Síndrome de Corazón

Izquierdo Hipoplásico;

Cirugía de Norwood;

Sobrevida 
asociada a la primera cirugía y sobrevida al año. Resultados: Se incluyeron 19 pacientes. La mayoría fueron recién nacidos de término y 13/19 de sexo femenino. Todos tenían diagnóstico prenatal de SCIH. Cinco pacientes presentaron malformaciones extracardiacas. De los 19 pacientes, 16 requirieron apoyo vasoactivo y 17 conexión a ventilación mecánica invasiva. Siete recibieron estímulo enteral, sin episodios de enterocolitis. La mediana de edad del traslado al centro cardioquirúrgico fue a los 8 días. Trece pacientes fueron sometidos a la cirugía de Norwood-Sano, con una letalidad de un $31 \%$ y una sobreviva al año de vida de $47 \%$. Conclusiones: El manejo neonatal de pacientes con $\mathrm{SCIH}$ es complejo y el enfrentamiento intensivo y multidisciplinario es fundamental. La letalidad alrededor de la primera etapa quirúrgica de nuestra cohorte es mayor que los reportes internacionales y podrían estar influyendo la ausencia de terapia fetal y la mayor incidencia de malformaciones extracardiacas.

\begin{abstract}
Hypoplastic Left Heart Syndrome (HLHS) is the most common form of functional single-ventricle heart disease. Despite improvements in medical and surgical care, morbidity and mortality remain high, especially around the first surgical stage. Objective: To describe the clinical characteristics of newborns with HLHS, seen at a perinatal referral center. Patients and Method: Retrospective descriptive study of all newborns with pre and/or postnatal diagnosis of HLHS between 2014 and 2019 in the Complejo Asistencial Dr. Sótero del Rio. Medical records were reviewed collecting demographic and perinatal variables, clinical evolution, mortality associated with the first surgery, and survival at one year. Results: Nineteen patients were included. Most were term newborns and 13/19 were female. All had a prenatal diagnosis. Five patients had associated extracardiac anomalies. 16/19 required vasoactive drugs and 17/19 used invasive mechanical ventilation. Seven patients received enteral feeding, without episodes of enterocolitis. The median age at transfer for surgery was 8 days. Thirteen patients underwent the Norwood procedure, with $31 \%$ of mortality, and $47 \%$ of survival at one year. Conclusions: Neonatal management of patients with HLHS is complex and the multidisciplinary approach is fundamental. Mortality around the first surgical stage of our cohort is greater than the international reports which could be influenced by the absence of fetal therapy and the higher incidence of extracardiac malformations.
\end{abstract}

Keywords:

Congenital Heart

Defects;

Hypoplastic Left Heart

Syndrome;

Norwood Procedure;

Survival Rate

\section{Introducción}

Los defectos cardíacos son las anomalías congénitas más frecuentes, con una incidencia de 4-10 por 1.000 recién nacidos vivos ${ }^{1,2}$. En Chile, la mortalidad por cardiopatías congénitas es la segunda causa de muerte en menores de 1 año y representa un tercio de las muertes por anomalías congénitas ${ }^{2}$. El diagnóstico prenatal de malformaciones cardíacas complejas ha modificado significativamente su pronóstico ${ }^{3}$.

El Síndrome de Corazón Izquierdo Hipoplásico (SCIH) engloba un espectro de cardiopatías congénitas complejas que se caracterizan por el desarrollo anormal de las estructuras del corazón izquierdo, cuyo factor común es un ventrículo izquierdo incapaz de sostener la circulación sistémica ${ }^{4-7}$. Mantienen una adecuada concordancia aurículo ventricular y ventrículo arterial, así como un septum interventricular intacto. Frecuentemente se acompaña de diferentes grados de hipoplasia de la válvula mitral, aórtica y arco aórtico ${ }^{4-7}$. Corresponde a la forma más frecuente de ventrículo único, dando cuenta del 2-3\% de todas las cardiopatías congénitas $s^{2,7}$. Sin una corrección quirúrgica su evolución es fatal, con una letalidad de un $95 \%$ antes del mes de vida ${ }^{7,8}$.

Durante los últimos 40 años se han llevado a cabo múltiples avances tanto médicos como quirúrgicos que han mejorado significativamente la sobrevida de estos pacientes ${ }^{7}$. Desde 1983 se describen 3 etapas quirúrgicas. La primera se lleva a cabo durante el período neonatal, siendo la de mayor letalidad (10-30\%), con tres alternativas: cirugía de Norwood con Shunt de Blalock Taussig modificado, cirugía de Norwood con conducto de Sano o un procedimiento híbrido (cerclaje de la arteria pulmonar e implante de un stent a nivel duc$\mathrm{tal})^{7,8,9}$. La segunda etapa corresponde a la anastomosis de Glenn, que se realiza a los 4-6 meses de vida y que tiene una letalidad perioperatoria entre el $2-5 \%{ }^{7,8,9}$. La última etapa es la cirugía de Fontan donde se completa la anastomosis cavo-pulmonar total, que se realiza alrededor de los 3-4 años de vida, con una letalidad de un $3-5 \% \%^{7,8,9}$. A pesar de los avances realizados, la sobrevida de estos pacientes no supera el $50-57 \%$ a los 5 años de vida ${ }^{9,10}$. 
En sus primeros días de vida, los pacientes requieren monitorización estricta del delicado balance entre el flujo pulmonar y sistémico ${ }^{7,8}$. Tras el descenso fisiológico de la resistencia vascular pulmonar, aumenta el flujo pulmonar que dependiendo de su cuantía, puede producir un daño permanente en su vasculatura e hipodébito sistémico secundario ${ }^{7,8}$. El manejo neonatal previo a la primera etapa quirúrgica se basa en la mantención de la permeabilidad ductal mediante el uso de prostaglandina E1 (PGE1) y el reconocimiento precoz de signos de hiperflujo pulmonar con hipodébito sistémico ${ }^{7,8}$. Existen diversas estrategias para aumentar la resistencia vascular pulmonar y de esta forma regular la cantidad de flujo pulmonar, que incluyen la sedación, conexión a ventilación mecánica invasiva (VMI) con presión positiva al final de la espiración (PEEP) elevada, frecuencias respiratorias bajas y eventual uso de mezcla de gases para disminuir la fracción inspirada de oxígeno bajo $21 \%{ }^{7,8}$. Para mantener una adecuada entrega de oxígeno a los tejidos se debe optimizar la volemia y mantener un hematocrito elevado $^{7,8}$. Pueden requerir además de apoyo inotrópico $^{7,8}$.

El Complejo Asistencial Dr. Sótero del Río (CASR) cuenta con un programa de manejo de cardiopatías congénitas desde el año $1991^{11}$. Actúa como centro de derivación perinatal de pacientes portadores de cardiopatías diagnosticados en el período fetal y/o neonatal provenientes de los Servicios de Salud de Iquique, Maule, Reloncaví, Chiloé y Metropolitano Sur Oriente. El paciente es evaluado en el período prenatal en el CASR, nace en dicho centro, es estudiado y estabilizado para ser derivado posteriormente al Hospital Clínico de la Pontificia Universidad Católica (HCUC) para realizarse la primera etapa quirúrgica. Posteriormente continúan el seguimiento en el hospital de base que le corresponde y las restantes etapas quirúrgicas se evalúan y realizan en el HCUC.

Considerando la complejidad del manejo médico y quirúrgico de pacientes con esta cardiopatía, es importante conocer las características de los pacientes desde el momento del diagnóstico prenatal, la evolución neonatal y postoperatoria de la primera cirugía, con el fin de evaluar en conjunto posibles aspectos a mejorar en la calidad de atención y entregar mayor información a los padres acerca de esta patología. El objetivo del presente estudio es describir las características perinatales, demográficas, evolución neonatal prequirúrgica, letalidad de la primera cirugía y sobrevida al año de vida de pacientes con SCHI atendidos en la Unidad de Obstetricia-Ginecología y Neonatología del CASR durante los últimos 5 años. El estudio se enfocará en el diagnóstico y evaluación fetal, aspectos obstétricos y en el manejo sólo durante el período neonatal hasta la primera cirugía.

\section{Pacientes y Método}

Estudio descriptivo de una cohorte retrospectiva de pacientes con diagnóstico de SCIH nacidos en el CASR entre enero del 2014 y enero del 2019. Se incluyeron todos los portadores de ventrículo izquierdo hipoplásico con concordancia auriculo-ventricular, ventrículo arterial y septum interventricular intacto, con diagnóstico prenatal de $\mathrm{SCIH}$, con confirmación postnatal y aquellos que sólo tuvieron diagnóstico postnatal, sin exclusiones.

Para la obtención de los datos prenatales se revisó el registro computacional de ecografías (Software Viewpoint) y base de datos de genética (Laboratorio de CitoGenética). Se revisaron las fichas clínicas de las madres para la obtención de datos de parto, estudios invasivos o procedimientos realizados durante el embarazo. En el caso de los recién nacidos (RN) se obtuvieron datos del registro de la ficha clínica durante su hospitalización y ficha ambulatoria. Se obtuvieron datos demográficos, registros ecocardiográficos, de ecografías cerebrales, estudio de malformaciones asociadas y evolución hospitalaria neonatal prequirúrgica, edad de traslado al HCUC para cirugía, tipo de cirugía y letalidad asociada.

Las variables numéricas se describieron como mediana, rango intercuartil (RIC) y las variables categóricas se expresaron en fracciones.

El estudio fue aprobado por el Comité de Ética en Investigación del Servicio de Salud Metropolitano Sur Oriente.

\section{Resultados}

Se reclutaron 19 pacientes con SCIH. Los datos demográficos se describen en la tabla 1 . De los 9 nacidos por cesárea, $7 / 9$ pacientes fueron por cesárea electiva por indicación obstétrica materna o fetal (3 por cicatriz de cesárea anterior, 2 por distocia de presentación fetal, 1 por la presencia de cardiopatía fetal y 1 por restricción de crecimiento intrauterino severo más oligohidroamnios) y $2 / 9$ correspondieron a cesáreas de urgencia. Dos pacientes presentaron un peso de nacimiento bajo los $2.500 \mathrm{~g}$.

Del total de los RN, 15/19 tuvieron un cariograma normal (pre y/o postnatal), en 4 no se realizó estudio. El estudio con FISH o MLPA del cromosoma 22q11 se realizó de forma postnatal en 11/19 pacientes y en 1 prenatal, todos sin microdeleción. Dentro del estudio de otras malformaciones, sólo un caso presentó alteración con agenesia del cuerpo calloso y septum pellucidum en la ecografía cerebral. Siete pacientes fueron estudiados con ecografía abdominal, de las cuales 6 fueron normales y 1 caso presentó riñón derecho intra- 
pélvico displásico. Con los estudios antes mencionados se encontró que 5/19 (26\%) de los RN presentaron malformaciones extracardíacas. En la tabla 1 se describen las malformaciones extracardíacas asociadas en 5 casos y las variantes cardíacas. Dos pacientes presentaron signos clínicos y ecocardiográficos de obstrucción al retorno venoso pulmonar secundario a un defecto interauricular restrictivo. Seis pacientes presentaron una aorta ascendente diminuta definida por un diámetro igual o menor a $2 \mathrm{~mm}$.

La descripción de la evolución neonatal previo a la primera cirugía se detalla en la tabla 2. Todos los pacientes recibieron PGE1, iniciadas durante el primer día de vida. Requirieron apoyo con drogas vasoactivas (DVA) 16/19 pacientes, determinado por: hipotensión (9/16), signos de hipodébito sistémico (4/16), hipertensión pulmonar persistente secundaria a hernia diafragmática congénita y sepsis $(1 / 16)$ e insuficiencia cardíaca congestiva (1/16). En relación al soporte ventilatorio, 17/19 requirieron conexión a VMI, que se inició desde las pocas horas de vida hasta los 8 días de vida. Las causas de conexión a VMI fueron: desaturación persistente, apneas, dificultad respiratoria, signos de insuficiencia cardíaca, depresión respiratoria secundaria a sedación y para manejo de hiperflujo pulmonar.

Todos los pacientes recibieron nutrición parenteral. Previo a la cirugía se inició estímulo enteral en 7 pacientes, con una mediana de inicio a los 4 días de vida (rango: 2 a 8 días). No hubo casos de enterocolitis necrotizante.

La mediana de edad del traslado para corrección quirúrgica fue de 8 días (rango: 4 y 18 días). La evolución quirúrgica y letalidad se describen en la figura 1. Cinco de los diecinueve pacientes quedaron fuera de alcance quirúrgico previo a la primera cirugía, 2 casos fueron excluidos por obstrucción al retorno venoso pulmonar, un caso por la asociación con un riñón displásico, uno por asociación con hernia diafragmática congénita derecha y otro por microcefalia y bajo peso de nacimiento. Además, previo a la primera etapa quirúrgica falleció un paciente por shock séptico refractario. La letalidad prequirúrgica total fue de un $32 \%$.

De los 13 pacientes sometidos a la primera cirugía (cirugía de Norwood-Sano), 1 falleció dentro de los primeros 30 días posterior a la cirugía (1 día post cirugía estando en ECMO) y 3 posterior a los 30 días y previo a la segunda intervención. La letalidad total en torno a la primera cirugía fue de un $31 \%$. Dos pacientes posterior a la primera cirugía fueron declarados fuera del alcance quirúrgico, un paciente con displasia septo-óptica que evolucionó con compromiso neurológico significativo que no había fallecido al momento del estudio y un segundo paciente con diagnóstico de atresia de vías biliares, que falleció posterior a los 30 días de realizada la cirugía de Norwood-Sano.
Tabla 1. Características demográficas, clínicas y variantes anatómicas cardíacas

\begin{tabular}{|c|c|}
\hline Características & $\mathrm{n}=19$ \\
\hline Sexo masculino, $\mathrm{n}$ & 6 \\
\hline \multicolumn{2}{|l|}{ Región de procedencia, $n$} \\
\hline Metropolitana & 12 \\
\hline$x$ & 3 \\
\hline VII & 4 \\
\hline Diagnóstico prenatal de CC, $\mathrm{n}$ & 19 \\
\hline $\begin{array}{l}\text { Edad gestacional de diagnóstico prenatal } \\
\text { en semanas, mediana (RIC) }\end{array}$ & $31(27,7-34,5)$ \\
\hline $\begin{array}{l}\text { Edad gestacional al nacer en semanas, } \\
\text { mediana (RIC) }\end{array}$ & $38(37-38)$ \\
\hline Peso de nacimiento en gramos, mediana (RIC) & $2.925(2.625-3.363)$ \\
\hline Parto vaginal, $n$ & 10 \\
\hline Apgar 1 min, mediana (RIC) & $8(2-9)$ \\
\hline Apgar 5 min, mediana (RIC) & $8(4-9)$ \\
\hline \multicolumn{2}{|l|}{ Malformaciones o patologías extracardiacas, n } \\
\hline Total & 5 \\
\hline HDC & 1 \\
\hline Neurológica & 2 \\
\hline Microcefalia & 1 \\
\hline Displasia septo-óptica & 1 \\
\hline Monorreno funcional & 1 \\
\hline Atresia vías biliares* & 1 \\
\hline \multicolumn{2}{|l|}{ Variante anatómica cardíaca, n } \\
\hline Estenosis mitral - estenosis aórtica & 6 \\
\hline Estenosis mitral - atresia aórtica & 3 \\
\hline Atresia mitral - estenosis aórtica & 1 \\
\hline Atresia mitral - atresia aórtica & 9 \\
\hline
\end{tabular}

CC: cardiopatía congénita, RIC: rango intercuartil, HDC: hernia diafragmática congénita. *Diagnóstico realizado posterior a la cirugía de Norwood-Sano.

Tabla 2. Tratamientos recibidos en el Servicio de Neonatología previo al traslado para cirugía

\begin{tabular}{ll}
\hline Tratamientos & \\
\hline Drogas Vasoactivas, n & $16(16 / 19)$ \\
Dopamina & $8(8 / 16)$ \\
Epinefrina & $3(3 / 16)$ \\
Dopamina + epinefrina & $3(3 / 16)$ \\
Dobutamina & $1(1 / 16)$ \\
Epinefrina + Milrinona & $1(1 / 16)$ \\
Prostaglandinas, n & $19(19 / 19)$ \\
Ventilación Mecánica Invasiva, n & $17(17 / 19)$ \\
Edad de traslado a corrección Quirúrgica, mediana (RIC) & $8(5,8-11)$
\end{tabular}

RIC: rango intercuartil. 
Figura 1. Etapas quirúrgicas y letalidad asociada. Nota: Porcentaje es en relación al número total de pacientes

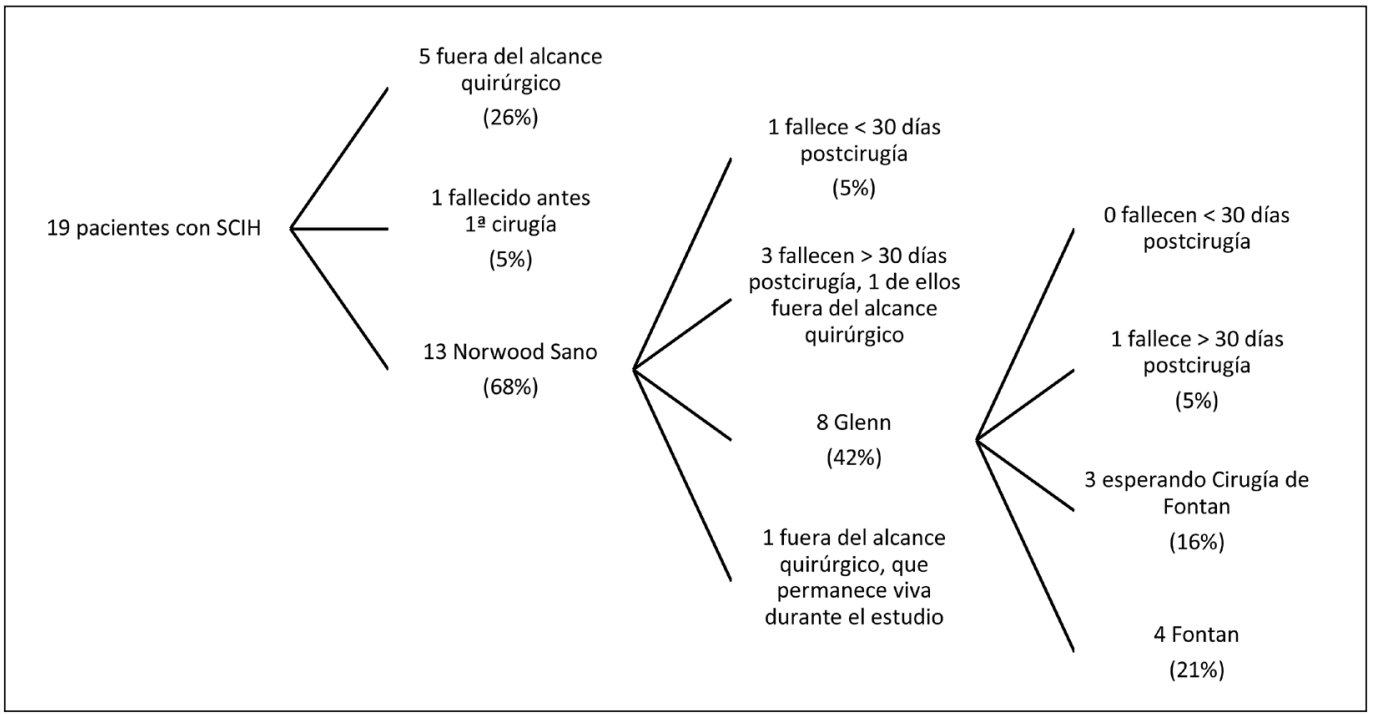

Ocho fueron sometidos a la cirugía de Glenn (42\%) y cuatro pacientes completaron la anastomosis cavopulmonar total (21\%). La sobrevida al año de vida en esta serie fue de un $47 \%$.

\section{Discusión}

El SCIH representa un desafío diagnóstico y terapéutico, que involucra tanto a especialistas en perinatología, neonatología, cardiología infantil y cardiocirugía, destacando la importancia del trabajo multidisciplinario y la coordinación de las distintas especialidades involucradas en el manejo de pacientes con esta patología. Esta serie clínica refuerza que los pacientes con diagnóstico de SCIH siguen presentando una alta morbilidad y letalidad a pesar de los avances en técnicas quirúrgicas y médicas ${ }^{9,10}$.

La detección prenatal depende de la disponibilidad de equipos de ecografía, de la capacitación y derivación apropiada a especialistas ${ }^{12}$. Actualmente, el diagnóstico prenatal de malformaciones cardíacas congénitas fetales en manos expertas es del $90-100 \%{ }^{3}$. Sin embargo, el acceso a este examen es limitado en muchos países, lo cual disminuye la pesquisa a rangos del 12-75\% dependiendo de las características de salud del país. Hoy en día, la mayoría de las embarazadas tienen una ecografía obstétrica general que incluye la evaluación de las cuatro cámaras cardíacas ${ }^{3}$. En esta cohorte retrospectiva, todos los pacientes tuvieron diagnóstico prenatal, lo que puede ser explicado porque nuestra institución corresponde a un centro de derivación de cardiopatías congénitas en el período perinatal de algunas regiones de nuestro país.
La detección prenatal de fetos con SCIH es importante para un eventual manejo fetal, consejo familiar, consejo genético y para optimizar el manejo postna$\mathrm{tal}^{5,8}$. En la actualidad, a nivel internacional existen alternativas de terapia fetal (septostomía de Rashkind, dilatación de la válvula aórtica con balón en forma intrauterina) con resultados variables, que no han sido desarrolladas en nuestro país ${ }^{5}$. El diagnóstico prenatal permite la coordinación multidisciplinaria para que estos pacientes nazcan en un centro con experiencia en el manejo de esta patología, permitiendo optimizar la condición neonatal preoperatoria, disminuyendo la morbilidad y letalidad ${ }^{5,8}$. Se ha descrito que los pacientes con diagnóstico prenatal de SCIH presentan menor incidencia de falla cardíaca y de otros órganos, menor conexión a VMI, uso de antimicrobianos, necesidad de apoyo vasoactivo y de cirugía de urgencia ${ }^{12}$.

Las anomalías cromosómicas están presentes en aproximadamente un $20 \%$ de los casos de cardiopatías congénitas ${ }^{13}$. Si bien la mayoría de los casos son esporádicos, existen asociaciones con algunos síndromes genéticos como Síndrome de Turner, Trisomía 13 y $18^{5}$. La evaluación genética prenatal es muy importante ya que ayuda a determinar pronóstico y posibilidad quirúrgica postnatal ${ }^{5}$. En nuestro hospital, se ofrece diagnóstico genético prenatal a todas las pacientes embarazadas de fetos que tienen esta patología, pero no todos los padres acceden a su realización dado los riesgos de la amniocentesis.

La asociación con malformaciones extracardíacas en esta cohorte fue mayor que lo publicado en la literatura internacional $(26 \% \text { versus } 6 \%)^{14}$. Esto podría deberse a que en otros países esta patología constituye una causa de interrupción voluntaria del embara- 
zo, por lo que los pacientes que nacen corresponden a aquellos que no presentan otras malformaciones asociadas.

En cuanto a la vía de parto, los datos reportados por grandes series internacionales no demuestran beneficios en pacientes nacidos por cesárea electiva versus parto vaginal ${ }^{15}$. Peterson reportó un análisis retrospectivo de pacientes con diagnóstico prenatal de SCIH comparando los resultados neonatales según la vía de parto. Concluyen que los pacientes nacidos por cesárea electiva tienen una edad gestacional menor y gas de cordón con menor $\mathrm{pH}$. Se ha postulado que esto se debería a que la cesárea electiva tiene efectos negativos a nivel de la adaptación pulmonar extrauterina, afectando el gasto cardíaco ${ }^{15}$. El análisis de esta cohorte en cuanto a la vía de parto muestra que la tasa de cesárea fue discretamente más elevada en comparación con los datos nacionales de cesárea en hospitales públicos (47 vs $37 \%)^{16,17}$. Al analizar las razones de las indicaciones de cesárea, en sólo una de las madres no hubo una causa obstétrica justificada. El protocolo de interrupción de nuestra institución del embarazo en caso de fetos con SCIH es idealmente a las 39 semanas de gestación, edad gestacional asociada a menor morbilidad y mejores resultados neonatales ${ }^{18}$.

Respecto al manejo neonatal previo a la primera cirugía, la tendencia actual es a minimizar los procedimientos invasivos. Si el paciente se mantiene estable, se debe tolerar cierto grado de hiperflujo pulmonar mientras se mantenga sin signos de hipoperfusión sistémi$\mathrm{Ca}^{7,8}$. En el presente estudio, la tasa de conexión a VMI fue elevada (89\%) en comparación a lo descrito en la literatura (70\%) y sólo en el $41 \%$ de los casos el motivo de conexión a VMI fue para manejo del hiperflujo pulmonar con hipodébito sistémico ${ }^{19}$. El uso de DVA en nuestro centro fue de un $84 \%$ que es comparable a lo reportado en la literatura $(30-88 \%)^{19,20,21,22}$. Para iniciar una DVA en estos pacientes, se deberían considerar los signos de mala perfusión sistémica (como oliguria, acidosis metabólica, hiperlactacidemia, etc) sumado a la presencia o no de hipotensión ${ }^{22}$.

El inicio del estímulo enteral es muy variable entre centros, con un rango entre el 56\%-93\% de los pacientes $^{23}$. Hay pocos reportes publicados acerca de la seguridad y tolerancia enteral en pacientes con cardiopatías ductus dependientes. Se suele postergar principalmente por el riesgo de presentar enterocolitis necrotizante $(\mathrm{ECN})$, sacrificando los conocidos beneficios de la alimentación con leche materna $(\mathrm{LM})^{24}$. Day y cols. evaluaron pacientes con cardiopatías congénitas ductus dependientes y la sospecha de ECN fue del $10 \%(\mathrm{n}=18)$ con sólo 2 casos confirmados ${ }^{24}$. Otro estudio retrospectivo realizado por Toms y cols., concluyó que el estímulo trófico puede ser seguro, bien tolerado e incluso con mejores resul- tados postquirúrgicos, con menos días de VMI y estadía hospitalaria ${ }^{25}$. En la actualidad, no existe evidencia que contraindique el inicio de aporte enteral en pacientes con $\mathrm{SCIH}$, especialmente si se cuenta con LM, si bien pareciera seguro hacerlo sólo en pacientes estables y en volúmenes pequeños. En nuestra institución el inicio de aporte enteral trófico (privilegiando leche materna por sobre fórmula) se realiza sólo en pacientes sin signos de hipodébito sistémico. En esta serie, 6 pacientes recibieron estímulo enteral, sin evidenciar episodios de ECN.

Si bien la cirugía cardíaca ha mejorado la sobrevida global, las secuelas del neurodesarrollo siguen siendo prevalentes ${ }^{26}$. Se describe alrededor de un 30$60 \%$ de alteraciones neurológicas ${ }^{26}$. Los factores que contribuyen a esto son el tiempo de hipoxia desde el período fetal, el uso de circulación extracorpórea y paro circulatorio en hipotermia durante la cirugía, la presencia de múltiples cirugías, tiempos prolongados de hospitalización y condicionantes genéticos además de los socioeconómicos ${ }^{26}$. En opinión de los autores, es importante crear un programa de seguimiento multidisciplinario formal, que involucre a todas las subespecialidades para el manejo integral del paciente, con estrategias nuevas como telemedicina. Esto último considerando que los pacientes pertenecen a distintas ciudades del país y continúan sus controles en sus centros de base, con el fin de conocer cuál fue su evolución a largo plazo.

En esta serie la sobrevida al año de vida es más baja respecto a lo descrito en la literatura nacional, probablemente porque incluimos aquellos casos que quedaron fuera del alcance quirúrgico. La experiencia reportada en la literatura nacional corresponde a los resultados publicados el año 2016 de 76 pacientes con SCIH tratados en el Hospital Clínico de la Pontificia Universidad Católica (HCUC) entre los años 2000 y 2010. En este reporte describen una sobrevida de un $64 \%$ al año y un $57 \%$ a los 5 años de vida ${ }^{9}$. En cuanto a la letalidad de la primera etapa quirúrgica, nuestro estudio muestra un porcentaje mayor a lo descrito en la literatura $(31 \% \mathrm{v} / \mathrm{s} 16-20 \%)^{27,28}$. Esto podría deberse a varios factores, entre los cuales pueden estar la falta de disponibilidad de terapia fetal, la mayor incidencia de malformaciones extracardíacas que las series internacionales y la necesidad de traslado a otro centro hospitalario. Otro factor a destacar es que las cardiopatías congénitas mayores aisladas no están dentro de las patologías que incluye la ley de interrupción voluntaria del embarazo en nuestro país, a diferencia de datos europeos que muestran tasas de terminación del embarazo por cardiopatía congénita prenatal entre el $31-57 \%{ }^{29,30}$.

Durante los últimos años se han realizado esfuerzos para identificar a los pacientes de mayor riesgo 
de morbilidad y letalidad. Tabutt y cols. identificaron como factores de alto riesgo a los pacientes con bajo peso de nacimiento, portadores de anomalías genéticas y los que tuvieron necesidad de ECMO o presentaron un paro cardiorrespiratorio previo a la primera cirugía ${ }^{31}$. En el reporte nacional del HCUC, establecieron como factor de alto riesgo de mortalidad la presencia de una aorta ascendente diminuta y un retorno venoso pulmonar obstructivo ${ }^{9}$. En nuestra serie, de todos los pacientes que fallecieron durante el seguimiento, sólo uno no tenía alguno de los factores de riesgo descritos.

Una de las principales limitaciones de este reporte es el pequeño tamaño muestral. Sin embargo, consideramos importante conocer la realidad local para mejorar el manejo y eventualmente el pronóstico de nuestros pacientes, además de describir los resultados de un centro a nivel local.

\section{Conclusión}

El trabajo multidisciplinario y la coordinación de las distintas especialidades (perinatólogos, neonatólogos, cardiólogos y cardiocirujanos) es fundamental en los RN con SCIH. El manejo neonatal es complejo $y$ debe estar enfocado en mantener un equilibrio entre la circulación pulmonar y sistémica, de forma de optimizar las condiciones en que los pacientes llegan a su primera etapa quirúrgica ${ }^{32}$. La letalidad alrededor de la primera etapa quirúrgica en nuestra cohorte retrospectiva es mayor que los reportes internacionales, lo que podría estar explicado en parte por la falta de disponibilidad de terapia fetal, la mayor incidencia de malformaciones extracardíacas y la necesidad de traslado a otro centro hospitalario.

\section{Responsabilidades Éticas}

Protección de personas y animales: Los autores declaran que los procedimientos seguidos se conformaron a las normas éticas del comité de experimentación humana responsable y de acuerdo con la Asociación Médica Mundial y la Declaración de Helsinki.

Confidencialidad de los datos: Los autores declaran que han seguido los protocolos de su centro de trabajo sobre la publicación de datos de pacientes.

Derecho a la Privacidad y Consentimiento Informado: Este estudio ha sido aprobado por el Comité de Ética de Investigación correspondiente, quien de acuerdo a las características del estudio ha eximido el uso del Consentimiento Informado.

\section{Conflicto de intereses}

Los autores declaran no tener conflicto de intereses.

\section{Agradecimientos}

Agradecemos al equipo de cardiología infantil del Hospital Clínico de la Universidad Católica por los datos aportados en cuanto a la primera cirugía cardíaca. También queremos agradecer a todo el equipo de salud de los Servicios de Neonatología y Cardiología Infantil, Centro de Investigación e Innovación en Medicina Materno-Fetal (CIMAF), Servicio de Obstetricia y Ginecología y Laboratorio de Citogenética del Complejo Asistencial Dr. Sótero del Río, los cuales colaboraron e hicieron posible la realización de este proyecto.

\section{Referencias}

1. Tutunji L, Thekrallah F, Basha A, et al. Prenatal detection of fetal heart disease at Jordan University Hospital: early experience in a developing country. Cardiol Young 2019;29(8):1072-7.

2. Guía Clínica Cardiopatías congénitas operables en menores 15 años, 2010, MINSAL,Chile.https://www.minsal.cl/ portal/url/item/720bfefe9le0d2ede040010 11 f010ff2.pdf. Consulta en Marzo 2020.

3. Acherman RJ, Evans WN. Chapter 16: Overview of Fetal Echocardiography. En: Practical Neonatal Echocardiography. McGraw Hill Education 2019;259-77.

4. Connor JA, Thiagarajan R. Hypoplastic left heart syndrome. Orphanet J Rare Dis 2007;2:23.

5. Feinstein JA, Benson DW, Dubin AM, et al. Hypoplastic left heart syndrome: current considerations and expectations. J Am Coll Cardiol. 2012;59(1):S1-42.

6. Rogers L, Pagel C, Sullivan I, et al. Interventions and Outcomes in Children With Hypoplastic Left Heart Syndrome Born in England and Wales Between 2000 and 2015 Based on the National Congenital Heart Disease Audit. Circulation. 2017;136:1765-7.

7. Yabrodi M, Mastropietro CW. Hypoplastic left heart syndrome: from comfort care to long-term survival. Pediatr Res. 2017;81(1-2):142-9.

8. Roeleveld PP, Axelrod DM, Klugman D, et al. Hypoplastic left heart syndrome: from fetus to fontan. Cardiol Young. 2018;28(11):1275-88

9. Urcelay G, Arancibia F, Retamal J, et al. Síndrome de hipoplasia de corazón izquierdo: experiencia de 10 años de un programa de etapificación quirúrgica. Rev Chil Pediatr. 2016;87(2):121-8.

10. Fixler DE, Nembhard WN, Salemi JL, et al. Mortality in First 5 Years in Infants With Functional Single Ventricle Born in Texas, 1996 to 2003. Circulation. 2010;121:644-50.

11. Castillo ME, Toro L, Zelada P, et al. Calidad de vida en pacientes portadores de cardiopatías congénitas. Rev Chil Cardiol. 2010;29:57-67.

12. Wacker-Gussmann A, Ehringer-Schetitska D, Herceg-Cavrak V, et al. Prevention of delayed diagnosis in congenital heart disease. Cardiol Young 2019;29(5):730-1.

13. Hopkins MK, Dugoff L, Kuller JA. Congenital Heart Disease: Prenatal Diagnosis and Genetic Associations. Obstet Gynecol Surv. 2019;74(8):497-503. 
14. Öhman A, El-Segaier M, Bergman G, et al. Changing Epidemiology of Hypoplastic Left Heart Syndrome: Results of a National Swedish Cohort Study. J Am Heart Assoc. 2019;8(2):e010893.

15. Peterson AL, Quartermain MD, Ades A, et al. Impact of Mode of Delivery on Markers of Perinatal Hemodynamics in Infants with Hypoplastic Left Heart Syndrome. J Pediatr. 2011:159(1):64-9.

16. Guzmán E. Perfil epidemiológico de la cesárea en Chile en la década 2000-2010. Medwave 2012;12(3):e5331.

17. Carreño B, Castillo V, Aichele D, et al. Tasa de cesáreas según la clasificación de Robson: Análisis comparativo entre dos hospitales universitarios. Rev. chil. obstet. ginecol. 2018;83(4):415-25.

18. Costello JM, Polito A, Brown DW. Birth Before 39 Weeks' Gestation Is Associated With Worse Outcomes in Neonates With Heart Disease. Pediatrics 2010;126:e277-e284.

19. Azakie A, Merklinger SL, McCrindle BW, et al. Evolving Strategies and Improving Outcomes of the Modified Norwood Procedure: A 10-Year SingleInstitution Experience. Ann Thorac Surg. 2001;72:1349-53.

20. Dempsey E, EL-Khuffash A. Clinical Trials in Hemodynamic Support. Past, Present and Future. Clin Perinatol. 2020;47(3):641-52.
21. Tweddell JS, Hoffman GM, Mussatto $\mathrm{KA}$, et al. Improved survival of patients undergoing palliation of hypoplastic left heart syndrome: lessons learned from 115 consecutive patients. Circulation 2002;106(1):82-9.

22. Stieh J, Fischer G, Scheewe J, et al. Impact of preoperative treatment strategies on the early perioperative outcome in neonates with hypoplastic left heart syndrome. J Thorac Cardiovasc Surg. 2006;131(5):1122-9.e2.

23. Howley LW, Kaufman J, Wymore E, et al. Enteral feeding in neonates with prostaglandin-dependent congenital cardiac disease: international survey on currents trends and variations in practices. Cardiol Young 2012;22(2):1217.

24. Day TG, Dionisio D, Zannino D, et al. Enteral feeding in ductdependent congenital heart disease. J Neonata Perinatal Med. 2019;12(1):9-12.

25. Toms R, Jackson KW, Dabal RJ, et al. Preoperative Trophic Feeds in Neonates with Hypoplastic Left Heart Syndrome. Congenit Heart Dis. 2015;10(1):36-42.

26. Lloyd DF, Rutherford MA, Simpson JM, et al. The neurodevelopmental implications of hypoplastic left heart syndrome in the fetus. Cardiol Young. 2017;27(2):217-23.
27. Hamzah M, Othman HF, Elsamny E, et al. Clinical Outcomes and Risk Factors for In-Hospital Mortality in Neonates with Hypoplastic Left Heart Syndrome. Pediatr Cardiol. 2020 Apr;41(4):781-8.

28. Jacobs JP, Mayer Jr JE, Mavroudis C, et al. The Society of Thoracic Surgeons Congenital Heart Surgery Database: 2017 Update on Outcomes and Quality. Ann Thorac Surg. 2017;103(3):699-709.

29. Lytzen R, Vejlstrup N, Bjerre J, et al. Live-Born Major Congenital Heart Disease in Denmark: Incidence, Detection Rate, and Termination of Pregnancy Rate From 1996 to 2013. JAMA Cardiol. 2018;3(9):829-37.

30. Beroukhim RS, Gauvreau K, Benavidez OJ, et al. Perinatal outcomes after prenatal diagnosis of single-ventricle cardiac defects. Ultrasound Obstet Gynecol. 2015;45(6):657-63.

31. Tabbutt S, Ghanayem N, Ravishankar C, et al. Risk factors for hospital morbidity and mortality after the Norwood procedure: A report from the Pediatric Heart Network Single Ventricle Reconstruction trial. J Thorac Cardiovasc Surg 2012;144(4):882-95.

32. Fuller $\mathrm{S}$, Ghanayem N, Chai P, et al. Neonatal surgical reconstruction and peri-operative care for hypoplastic left heart syndrome: current strategies. Cardiol Young. 2011;21(2):38-46. 